\title{
Plasmodium Falciparum Phenotypic and Genotypic Resistance Profile During the Emergence of Piperaquine Resistance From Royal Thai Army and Civilian patients in Northeastern Thailand
}

\author{
Nonlawat Boonyalai ( $\square$ nonlawatb.fsn@afrims.org ) \\ Armed Forces Research Institute of Medical Sciences \\ Chatchadaporn Thamnurak \\ Armed Forces Research Institute of Medical Sciences
}

Piyaporn Sai-ngam

Armed Forces Research Institute of Medical Sciences

Winita Ta-aksorn

Armed Forces Research Institute of Medical Sciences

Montri Arsanok

Armed Forces Research Institute of Medical Sciences

Nichapat Uthaimongkol

Armed Forces Research Institute of Medical Sciences

Siratchana Sundrakes

Armed Forces Research Institute of Medical Sciences

Sorayut Chattrakarn

Armed Forces Research Institute of Medical Sciences

Chaiyaporn Chaisatit

Armed Forces Research Institute of Medical Sciences

Chantida Praditpol

Armed Forces Research Institute of Medical Sciences

Watcharintorn Fagnark

Armed Forces Research Institute of Medical Sciences

Kirakarn Kirativanich

Armed Forces Research Institute of Medical Sciences

Suwanna Chaorattanakawee

Mahidol University

Pattaraporn Vanachayangkul

Armed Forces Research Institute of Medical Sciences

Paphavee Lertsethtakarn

Armed Forces Research Institute of Medical Sciences

Panita Gosi

Armed Forces Research Institute of Medical Sciences

\section{Darunee Utainnam}

Royal Thai Army Component, Armed Forces Research Institute of Medical Sciences

Wuttikon Rodkvamtook

Royal Thai Army Component, Armed Forces Research Institute of Medical Sciences

Worachet Kuntawunginn

Armed Forces Research Institute of Medical Sciences

Brian A. Vesely

Armed Forces Research Institute of Medical Sciences

Michele D. Spring

Armed Forces Research Institute of Medical Sciences 
Mark M. Fukuda

Armed Forces Research Institute of Medical Sciences

Charlotte Lanteri

Walter Reed Army Institute of Research.

\section{Douglas Walsh}

Department of Dermatology, Syracuse, VA Medical Center

\section{David L. Saunders}

U.S. Army Research Institute of Infectious Diseases

\section{Philip L. Smith}

Walter Reed Army Institute of Research.

\section{Mariusz Wojnarski}

Armed Forces Research Institute of Medical Sciences

\section{Narongrid Sirisopana}

Royal Thai Army Component, Armed Forces Research Institute of Medical Sciences

\section{Norman C. Waters}

Armed Forces Research Institute of Medical Sciences

Krisada Jongsakul

Armed Forces Research Institute of Medical Sciences

Jariyanart Gaywee

Royal Thai Army Component, Armed Forces Research Institute of Medical Sciences

\section{Research Article}

Keywords: Artemisinin resistance, Chloroquine resistance transporter, Malaria, Multidrug resistance, Piperaquine resistance, Plasmepsin, Plasmodium falciparum, military, border, Thailand

Posted Date: April 7th, 2021

DOI: https://doi.org/10.21203/rs.3.rs-379153/v1

License: @ (1) This work is licensed under a Creative Commons Attribution 4.0 International License. Read Full License 


\section{Abstract}

Malaria remains a public health problem in Thailand, especially along its borders where highly mobile populations can contribute to persistent transmission. This study aimed to determine resistant genotypes and phenotypes of 112 Plasmodium falciparum isolates from patients along the Thai-Cambodia border during 2013-2015. The majority of parasites harbored pfmdr1-Y184F mutation. A single pfmdr1 copy number, had CVIET haplotype of amino acids 72-76 of pfcrt and no pfcytb mutations. All isolates had a pfk 13 point mutation (R539T, R539l, and C580Y), and increased ring-stage survival assay (except R539I). Multiple copies of pfpm2 and pfcrt-F145I were first detected in 2014 (12.8\%) and increased to 30.4\% in 2015. Parasites containing either multiple pfpm2 copies with and without pfcrt-F145I or a single pfpm 2 copy with pfcrt-F145I exhibited the elevated $\mathrm{IC}_{90}$ values of piperaquine. Antimalarials were detected in 21 samples (18.8\%) by mass spectrometer, and 12 samples (10.7\%) by bioassay. Collectively, the emergence of these resistance patterns mirrored the reports of dihydroartemisinin-piperaquine treatment failures in the adjacent province of Cambodia, Oddar Meanchey, suggesting a migration of parasites across the border. As malaria elimination efforts ramp up in Southeast Asia, host nations militaries and other groups in border regions must be a focus for interventions

\section{Introduction}

An estimate of 229 million malaria cases occurred worldwide in $2019^{1}$ and artemisinin-based combination therapies (ACT) have been the first-line drug treatment for uncomplicated Plasmodiumfalciparum infection for most malaria endemic areas; however, resistance to both artemisinin (ART) and its partner drugs has increased over the years at a pace requiring intensive surveillance and monitoring. The first case reports of ART resistance emerged in Thailand in the late $1990 \mathrm{~s}^{2}$ and in 2003-2005. Thai Ministry of Public Health (MoPH) and Armed Forces Research Institute of Medical Sciences (AFRIMS) studies began to suggest that first-line ACT regimens (2day artesunate (AS) plus mefloquine (MQ)) were failing along both sides of the Thai-Cambodia border near Trat, Thailand and Pailin, Cambodia $^{3}$. Militaries and other mobile forest goers in these border areas constitute a population at risk for succumbing to multi-drug resistant malaria as well as facilitating transmission, necessitating targeted control strategies if the Greater Mekong Subregion (GMS) is to achieve malaria elimination. Much of the research conducted at AFRIMS has mil-to-mil engagement with Thai and Cambodian armed forces, which allows for clinical and surveillance studies to be conducted in these high risk populations, resulting in interventions tailored to the military and shared with the $\mathrm{MoPH}$

Following the case reports of ACT failure, the Artemisinin Resistance: Confirmation, Characterization and Planning for Containment Project (ARC) in 2006 showed a delayed clearance resistance phenotype in Cambodia along the Thai border which continued to spread across mainland Southeast Asia ${ }^{4-9}$. But it was not until 2014 that an ART resistance molecular marker was identified with mutations in the propeller domain of $P$. falciparum Kelch-13 gene (pfk13) (PF3D7_1343700) ${ }^{10-13}$, which can be confirmed by the ring-stage survival assay (RSA) ${ }^{10,14}$ and/or delayed parasite clearance times in clinical trials ${ }^{4,5}$. Multiple copies of $P$. falciparum multi drug resistance 1 (pfmdr1) (PF3DF_0523000) is a well-established marker for MQ resistance ${ }^{15,16}$, while the pfmdr1 single-nucleotide polymorphisms (SNPs) have been associated with modulation of parasite tolerance or susceptibility to several antimalarial drugs including quinine $(\mathrm{QN})$, amodiaquine $(\mathrm{AQ})$, chloroquine (CQ), MQ and lumefantrine (LUM $)^{17}$. The main pfmdr1 SNPs associated with drug resistance include N86Y, Y184F, S1034C, and N1024D ${ }^{18-23}$. The wild-type N86 in pfmdr1 has been associated with increased tolerance to the artemether (ATM) and LUM ${ }^{18,23,24}$, while parasites harboring the N86Y mutation exhibit an increased susceptibility to MQ ${ }^{25}$. An ACT alternative to AS-MQ is dihydroartemisinin-piperaquine (DHA-PPQ) and the first PPQ resistance markers identified were multiple copies of $P$. falciparum plasmepsin 2 (pfpm2) (PF3D7_1408000) ${ }^{26-29}$, followed by mutations in $P$. falciparum chloroquine resistance transporter ( $p f c r t$ ) (PF3D7_0709000) downstream of the 4-aminoquinoline resistance locus (positions 72-76 with K76T) ${ }^{30-33}$, and the E415G mutation in P. falciparum exonuclease (pfexo) (PF3D7_1362500) ${ }^{26,27,34}$. PPQ resistance can be characterized by the elevated $\mathrm{IC}_{90}$ values $^{35}$ and PPQ survival assay (PSA) with a survival rate of more than $10 \%$, defining a PPQ resistant phenotype ${ }^{30}$. Atovaquoneproguanil (ATQ-PG) is not an ACT but ATQ resistance subsequently emerged which was linked to specific mutations in P. falciparum mitochondrial cytochrome B (pfcytb) (AY282930.1) in particular the mutation at positions 258 and 268 $36-38$.

Twenty years after the first ACT failures, there are now descriptions of the spread of a single multidrug resistant malaria parasite lineage (PfPailin) across the eastern $\mathrm{GMS}^{39,40}$. Efforts in mapping parasite population structure and gene flow can assist in understanding and predicting the spreading pattern of resistance. Military populations at border areas who are deployed to endemic areas should be integrated in surveillance and monitoring efforts as they are on the front-lines of malaria transmission. Here we report resistance characteristics of $P$. falciparum clinical isolates collected from Thai soldiers and civilian patients between 2013 and 2015 
and show the trends in drug resistance and spread. At that time, the national treatment guidelines in Thailand recommended AS-MQ for $P$. falciparum malaria. During this same period, we reported dramatic loss of efficacy of DHA-PPQ ( $54 \%$ treatment failure) in a study that was conducted by AFRIMS in Anlong Veng, $12 \mathrm{~km}$ from the Thai border of Sisaket Province ${ }^{41}$. As the neighboring countries strive to achieve malaria elimination, there is ongoing concern about the risk of malaria transmission across borders, where treatment guidelines are different.

While there is a pressing need for new classes of antimalarials, intensive malaria surveillance to track drug resistance is required in high risk military populations to achieve malaria control so that these border foci of infections no longer pose a threat to elimination. Here, the investigation into in vitro drug susceptibility and molecular drug resistance marker profiling of $P$. falciparum isolates in patients presenting to Thai military health facilities is reported.

\section{Materials And Methods}

\section{Study setting, protocol and subjects}

This minimal risk surveillance study was open between July 2013-September 2015, enrolling Thai adults (aged 18 years and over) presenting with uncomplicated $P$. falciparum or $P$. falciparum/ $P$. vivax mixed-infections at military health facilities. The enrollment population included soldiers, border police, or their family members and other villagers located near the Royal Thai Army (RTA) health clinics in Sisaket and Surin Provinces, located in northeastern Thailand on the Thai-Cambodia border. Inclusion criteria were asexual parasitemia per rapid diagnostic test (RDT) or blood film, and no malaria infection or antimalarials taken within the past seven days. The protocol was approved by the Walter Reed Army Institute of Research Institutional Board, Institute for Development of Human Research Protection, and RTA Institutional Review Board. All research was performed in accordance with relevant guidelines and regulations. All study subjects provided written informed consent prior to participation. Goal enrollment was 50 malaria cases per year, balancing the number of isolates needed to characterize resistant genotypes/phenotypes with the local population size and expected incidence of cases that could be enrolled.

\section{Sample collection and preparation}

Patients diagnosed with P. falciparum infection at RTA clinics were subjected to peripheral venipuncture prior to treatment. Two microscopists examined Giemsa-stained peripheral blood smears for each volunteer to determine malaria species infection and parasite densities for blood stages. Venous blood samples were collected in EDTA tubes for DNA extraction and molecular characterization and in sodium-heparin tubes for ex vivo bioassay and in vitro drug sensitivity assay. Plasma was separated from blood, frozen at $-80^{\circ} \mathrm{C}$, and infected packed red blood cells were cryopreserved. All blood and processed blood samples were stored at $-80 \mathrm{C}$ and transported in dry ice to AFRIMS for molecular characterization, ex vivo bioassay, and in vitro culture adaptation and drug sensitivity testing.

\section{Molecular markers of malaria drug resistance}

Parasite genomic DNA was extracted from $200 \mu \mathrm{L}$ of EDTA whole blood by using EZ1 DNA blood kit with an automated EZ1 Advanced $\mathrm{XL}$ purification system as per the manufacturer's instructions. The DNA was stored at $-20^{\circ} \mathrm{C}$. T100TM Thermal Cycler (Bio-Rad Laboratories) was employed to evaluate resistance makers including, P. falciparum kelch13 propeller domain (pfk13) (PF3D7_1343700), pfcrt SNP F145I (KM288867.1), and pfcytb SNPs (AY282930.1) ${ }^{10,42}$. ABI 7500 Real-time PCR system (Applied Biosystems) was employed to characterize pfcrt SNPs (72-76) and pfmdr1 SNPs (Positions 86, 184, 1034, 1042). Primers used to identify pfkelch13, pfcytb, pfcrt and pfmdr1 SNPs are shown in Tables S1 and S2.

\section{Copy number variation assay}

To determine copy numbers of $p f m d r 1$ and pfpm 2 genes, real-time quantitative PCR (qPCR) was performed on genomic DNA as previously described ${ }^{15,28,43}$ with some modifications. For pfmdr1, amplification reactions were performed according to the TaqMan Real-time PCR methods using ABI 7500 Real-time PCR system (Applied Biosystems) with $200 \mathrm{nM}$ of each forward and reverse primer (Table S2) and 2 ng of DNA template while Rotor-Gene Q (QIAGEN, Valencia, CA) using Type-it ${ }^{\circledR}$ HRM $^{\text {Tm }}$ kit was employed for $p f p m^{28}$. The primers and probes (Table S2) used were as previously described to amplify the following loci: pfmdr1 (PF3D7_0523000) and pfpm2 (PF3D7_1408000), respectively ${ }^{28}$. For the housekeeping gene, $\beta$-tubulin (PF3D7_1008700), â-tubulin forward and reverse primers were designed and used as a reference control for all experiments with the same validated PCR conditions as target primers. $P$. 
falciparum 3D7 and Dd2 were used as references for single and multiple copy numbers of $p f m d r 1$, respectively. All samples including the references clones were performed in duplicate. The average copy number values for each genes were calculated using $2^{-\Delta \Delta C t}$ method. Parasites with copy number greater than 1.5 copies for $p f m d r 1^{15}$ and 1.6 copies for $p f p m 2^{28}$ were interpreted to contain multiple copies of each gene.

\section{In vitro Culture adaptation and maintenance of parasites}

Of 112 collected samples, 86 samples were subjected for in vitro culture adaptation. The exclusion criteria of the in vitro culture adaptation were $P$. falciparum/P. vivax mixed infections, \% parasitemia $<0.05$, and ex vivo bioassay activity $>250 \mathrm{nM}$ (DHA equivalent). Culture adaptation of the parasites was performed using the modification method of Trager and Jensen ${ }^{44}$. Parasites were maintained in fresh human erythrocytes $\left(\mathrm{O}^{+}\right)$in RPMI-1640 medium (Sigma), containing 15\% $\mathrm{AB}^{+}$human serum (heat inactivated and pooled) and supplemented with $25 \mathrm{mM}$ HEPES, $25 \mathrm{mM}$ sodium bicarbonate, and $0.1 \mathrm{mg} / \mathrm{mL}$ gentamicin. Human blood products (erythrocytes and serum) were obtained from the Thai Red Cross. Culture flasks were gassed with $5 \% \mathrm{CO}_{2}, 5 \% \mathrm{O}_{2}, 90 \% \mathrm{~N}_{2}$ and incubated at $37^{\circ} \mathrm{C}$.

\section{In vitro 72 hours drug susceptibility by Histidine rich protein 2 (HRP2)}

Drug susceptibility test using HRP2-ELISA to measure $50 \%$ or $90 \%$ inhibitory concentration $\left(\mathrm{IC}_{50}\right.$ and $\left.\mathrm{IC}_{90}\right)$ was performed following

previously published methods ${ }^{45-47}$. Dried drug-coated plates containing antimalarial drugs as described in Chaorattanakawee et al,35,46 were used and in vitro drug susceptibility testing was carried out for control reference clones (W2, D6, C2B) (Malaria Research \& Reference Reagent Resource, Manassas, Vermont, USA) as described previously ${ }^{35}$. I $\mathrm{C}_{50} \mathrm{~S}$ and $\mathrm{IC}_{90} \mathrm{~S}$ were estimated by nonlinear regression analysis using GraphPad Prism version 6.0.

\section{Ring-stage survival assay (RSA)}

In vitro $\mathrm{RSA}_{0-3 \mathrm{~h}}$ was performed on 0-3 h post-invasion rings obtained from selected culture-adapted clinical isolates following published methods with slight modifications ${ }^{14,47}$. Parasite cultures were synchronized using $5 \%$ D-sorbitol and $75 \%$ Percoll to obtain 0 to 3 -h post-invasion rings which were adjusted to $0.5-1 \%$ starting parasitemia with a $2 \%$ hematocrit in culture media ( $0.5 \%$ Albumax RPMI 1640 with $2.5 \% \mathrm{AB}$ serum), and cultured in a 48-well microplate with $700 \mathrm{nM}$ DHA and $0.1 \%$ DMSO in separate wells. The culture plate was then incubated for 6 hours at $37^{\circ} \mathrm{C}$ in modular incubator chambers and gassed with $5 \% \mathrm{CO}_{2}, 5 \% \mathrm{O}_{2}$ and $90 \% \mathrm{~N}_{2}$. Cells were then washed once, resuspended in a drug-free medium, and cultured further for 66 hours. Susceptibility to DHA was assessed microscopically on thin films by estimating the percentage of viable parasites, relative to control (\% survival rate). For the controls, the $\mathrm{RSA}_{0-3 \mathrm{~h}}$ was also performed on $P$. falciparum reference clones W2, IPC-4884 and IPC-5202 (BEI Resources, NIAID, NIH, USA). A survival rate $>1 \%$ was deemed resistant for RSA.

\section{Ex vivo bioassay}

P. falciparum-based bioassay was carried out to measure the antimalarial activity of patient plasma identifying if study volunteers were likely to have recently taken antimalarial drugs. Plasma was prepared from blood collected on the screening day for evaluation according to previously published methods ${ }^{48,49}$. In brief, the complement-inactivated samples were serially diluted and applied in one column to $96-w e l l$ microplate at $50 \mu \mathrm{L} /$ well. Two columns with serial dilutions of spiked plasma were added to each plate as controls. In addition to the plates with unknown samples, one plate was dosed with six serial dilutions in duplicate of DHA (from 100 to 2.5 $\mathrm{ng} / \mathrm{mL}$ ). A suspension of $200 \mu \mathrm{L}$ of malaria parasite-infected red blood cells (W2 clone; $0.05 \%$ parasitemia with $>80 \%$ rings at a $1.7 \%$ hematocrit) was added to all wells. The microplates were placed into a chamber, flushed with a mixture of gas consisting of $5 \% \mathrm{CO}_{2}$, $5 \% \mathrm{O}_{2}$ and $90 \% \mathrm{~N}_{2}$, and plated into an incubator at $37^{\circ} \mathrm{C}$ for $72 \mathrm{~h}$. HRP2-ELISA was performed as described above.

\section{Liquid Chromatography-Tandem Mass Spectrometry (LC-MS/MS) Analysis}

To detect baseline antimalarials prior to treatment in the study population, plasma samples were extracted by using protein precipitation 2-fold volume of acetonitrile containing internal standard, then 1-minute vortex mixing, 10 minute-centrifuged, filtered supernatant with $0.22 \mu \mathrm{m}$ PTFE filter and then transferred to HPLC vial. The LC separation was performed on ACQUITY UPLC (Waters) coupled with tandem mass spectrometer (Xevo TQ-S, Waters) and eluted on Waters Acquity UPLC® BEH C18, $2.1 \times 50$ mm, $1.7 \mu \mathrm{m}$

Page 5/19 
column at a flow rate of $0.5 \mathrm{~mL} /$ minute in 8 minutes run time. Mobile phase consisted of (A) $5 \mathrm{mM}$ ammonium acetate pH 4.5 in water and (B) $5 \mathrm{mM}$ ammonium acetate $\mathrm{pH} 4.5$ in acetonitrile:methanol (50:50 v/v). The gradient started with $10 \% \mathrm{~B}$, raised to $98 \% \mathrm{~B}$ in 6 minutes and held at this composition for 0.5 minute, decreased to $10 \% \mathrm{~B}$ and re-equilibrated for 1 minute. Column temperature was set at $40^{\circ} \mathrm{C}$. Selective mass to charge $(\mathrm{m} / \mathrm{z}$ ) transition for each compound was monitored as follow: AS (407.4 > 261.23), DHA (307.13 > 261.26), MQ (379.1 > 321.0), carboxy primaquine (cMQ) (310.22 > 290.05), PG (256.1 > 171.9), cycloguanil (CYC) (251.95 > 194.9), primaquine (PQ) $(260.07>85.95)$, carboxy primaquine (cPQ) $(275.34>175.11)$, orthoquinone (OQ) $(260.2>147.08)$, stable isotopic labeled artesunate (SIL-AS) $(411.3>261.18)$, and stable isotopic labeled primaquine (SIL-PQ) $(264.35>86.15)$.

\section{Statistical analysis}

Statistical analysis was performed using GraphPad Prism version 6.0 (GraphPad Software, Inc., San Diego, CA, USA). The difference of data between groups was assessed by nonparametric Kruskal-Wallis, Mann-Whitney or Dunn's multiple comparison tests, as appropriate. Statistical significance was defined as a $P$ value $<0.05$.

\section{Results}

\section{Study population, demographic, and parasitological parameters}

In total, 117 individuals were enrolled but 5 individuals were excluded from analysis due to lack of $P$. falciparum on a blood smear in 4 patients and $P$. vivax monoinfection in 1 patient. Therefore, 112 individuals with uncomplicated $P$. falciparum were included in the analysis (Table 1). The median age of the participants was 23 years (IQR: 22-30.75) and most were male (98.2\%), in the military (83\%), and from Sisaket Province (96.4\%). Among rapid diagnostic test and/or microscopy positive malarial isolates, 108 samples (96.4\%) were confirmed by PCR as $P$. falciparum infections and 4 samples (3.6\%) as mixed $P$. falciparum and $P$. vivax infections. The geometric mean of parasitemia of the participants was 12,097 parasites $/ \mu \mathrm{L}$.

Table 1. Patient and parasitological characteristics of 112 participants.

\begin{tabular}{|c|c|c|c|c|}
\hline & 2013 & 2014 & 2015 & $\begin{array}{l}P \\
\text { value }\end{array}$ \\
\hline Number of cases & 27 & 39 & 46 & - \\
\hline Age (y), median (IQR) & $22(22-24)$ & $23(22-31)$ & $23(22-44)$ & 0.1210 \\
\hline Male:female, n (\%) & $27: 0(100: 0)$ & $39: 0(100: 0)$ & $44: 2(96: 4)$ & - \\
\hline Civilian:Military, n (\%) & $1: 26(4: 96)$ & $5: 34(13: 87)$ & $13: 33(28: 72)$ & - \\
\hline Weight (kg), median (IQR) & $60(56-67)$ & $62(60-71)$ & $60(58-67)$ & 0.698 \\
\hline Site location, Sisaket:Surin, n (\%) & $27: 0(100: 0)$ & $35: 4(90: 10)$ & $46: 0(100: 0)$ & - \\
\hline $\begin{array}{l}\text { Parasitemia (no./ } \mu \mathrm{L}), \text { geometric mean }(95 \% \\
\mathrm{Cl})\end{array}$ & $\begin{array}{l}11,963(6,698- \\
17,944)\end{array}$ & $\begin{array}{l}14,960(8,940- \\
25,035)\end{array}$ & $\begin{array}{l}10,704(7,158- \\
16,008)\end{array}$ & 0.320 \\
\hline P. falciparum, n (\%) & $27(100 \%)$ & $36(92 \%)$ & $45(98 \%)$ & - \\
\hline Mixed $P$. falciparum/ P. vivax, $\mathrm{n}(\%)$ & 0 & $3(8 \%)$ & $1(2 \%)$ & - \\
\hline
\end{tabular}

$P$ values calculated by the Kruskal-Wallis test

\section{Mutations in Pfmdr1, Pfcrt, Pfk13, and Pfcytb gene}

112 samples were evaluated for SNPs of pfmdr1, pfcrt (positions 72-76), pfcrt (position 145), pfk13, and pfcytb as molecular markers of drug resistance associated with MQ, CQ, PPQ, ART, and ATQ, respectively. Of four point mutations on pfmdr1, only the Y184F mutation (97.3\%) was detected (Table 2), suggesting the presence of two pfmdr1 haplotypes in the samples: NYSN and NFSN (representing amino acids positions $86,184,1034$, and 1042, respectively) with NFSN as the most prevalent genotype. Genotyping of pfcrt showed all mutant haplotypes at positions 72-76 (CVIET), which is associated with CQ resistance. Regarding the novel pfcrt mutation (F145I) associated with PPQ resistance, no F145I mutation was identified in 2013, but the F145I mutation of pfcrt was detected in 5 (12.8\%) samples and 14 (30.4\%) in 2014 and 2015, respectively. Concerning pfk13 as associated with ART resistance, 
three nonsynonymous mutations (R539T, R539l, and C580Y) in the k13 propeller domain were observed in 112 samples and C580Y was the predominant $p f k 13$ mutation, accounted for $84.8 \%$ of all pfk 13 mutations. The number of C580Y mutants increased from $2013(63 \%)$ to 2015 (100\%). There were no mutations detected in pfcytb for amino acid positions 258 and 268.

\section{Copy Number Variation of pfmdr1 and pfpm2}

Single pfmdr1 copies were present in nearly all of evaluable cases (107/112), only 5 samples had multiple copies of pfmdr1, found in samples collected in 2013 and 2014. Multiple pfpm2 copies were found in about half of evaluable cases (58/112), and the number of parasites harboring multiple pfpm 2 copies steadily rose from $7.4 \%$ to $78.3 \%$ from 2013 to 2015 . Of 5 samples containing multiple pfmdr 1 copies, all had single $p f p m 2$ copy and pfcrt F145 wild-type. All parasites harboring multiple $p f p m 2$ copies contained pfpk13C580Y mutation and of those samples, 13 samples (22.4\%) contained the novel pfcrt F145I mutation.

\section{Haplotype and Copy number variation (CNV) of $P$. falciparum isolates}

The parasite isolates were categorized into nine groups according to their genotypes of $p f m d r 1, p f k 13$, and $p f c r t$ in combination with their CNV of pfmdr1 and pfpm2 (Table S3). Overall, the most prevalent parasites were those in group III, containing pfk13-580Y alleles, multiple pfpm 2 copies, pfcrt-F145 alleles and pfmdr1 184F alleles with a single copy number. This was followed by parasites in group II which was the same as Group 3 except with pfpm2 single copy number.

The number of parasites in group II decreased from $55.6 \%$ to $13 \%$, while those in group III increased from $7.4 \%$ to $56.5 \%$. Parasites in group $\mathrm{Vl}$, containing the $p f c r t-145 \mathrm{I}$ and multiple $p f p m 2$ copies, were also found to be increasing over the study period. It was noted that parasites in group VII to IX, harboring the pfk13-539I/T alleles with no novel mutation on pfcrt, and pfmdr1-184F only held a single pfpm2 copy.

\section{Prevalence of molecular markers for ART-, MQ- and PPQ-Resistance}

With limited number of ACT options, we assessed if any parasites had all mutations for ART-, MQ-, and PPQ-resistance (pfk13 SNPs, pfmdr1 CNV, pfpm $2 \mathrm{CNV}$, and pfcrtF145I mutation). No tested parasites in this study carried all the aforementioned markers, although this is largely driven by having only 5 isolates with multiple copies of pfmdr1. If that marker is excluded, only $11.6 \%$ of the isolates (13/112) harbored pfk13-C580Y, multiple pfpm2 copies, and pfcrt-F145I (3 isolates in 2014 and 10 isolates in 2015). All of the 16 parasites with pfk13-R539T carried a single pfpm2 copy number with no pfcrt-F145I mutation but four of them had multiple pfmdr1 copies.

Table 2. Mutations of malaria resistance molecular markers of $P$. falciparum in Thailand between 2013 and 2015

\begin{tabular}{|c|c|c|c|c|c|c|c|c|c|c|c|c|}
\hline \multirow[t]{2}{*}{ Year } & \multirow{2}{*}{$\begin{array}{l}\text { No. of } \\
\text { tested }\end{array}$} & \multicolumn{4}{|c|}{ pfmdr1 } & \multicolumn{2}{|l|}{ pfert } & \multicolumn{3}{|l|}{ pfk13 } & \multicolumn{2}{|l|}{ pfcytb } \\
\hline & & N86Y & Y184F & S1034C & N1042D & $\begin{array}{l}\text { CVIET } \\
(72- \\
76)\end{array}$ & F145I & R539T & R539l & C580Y & I258M & Y268S \\
\hline 2013 & 27 & 0 & $\begin{array}{l}27 \\
(100 \%)\end{array}$ & 0 & 0 & $\begin{array}{l}27 \\
(100 \%)\end{array}$ & 0 & $\begin{array}{l}9 \\
(33.3 \%)\end{array}$ & $\begin{array}{l}1 \\
(3.7 \%)\end{array}$ & $\begin{array}{l}17 \\
(63.0 \%)\end{array}$ & 0 & 0 \\
\hline 2014 & 39 & 0 & $\begin{array}{l}36 \\
(92.3 \%)\end{array}$ & 0 & 0 & $\begin{array}{l}39 \\
(100 \%)\end{array}$ & $\begin{array}{l}5 \\
(12.8 \%)\end{array}$ & $\begin{array}{l}7 \\
(17.9 \%)\end{array}$ & 0 & $\begin{array}{l}32 \\
(82.1 \%)\end{array}$ & 0 & 0 \\
\hline 2015 & 46 & 0 & $\begin{array}{l}46 \\
(100 \%)\end{array}$ & 0 & 0 & $\begin{array}{l}46 \\
(100 \%)\end{array}$ & $\begin{array}{l}14 \\
(30.4 \%\end{array}$ & 0 & 0 & $\begin{array}{l}46 \\
(100 \%)\end{array}$ & 0 & 0 \\
\hline Total & 112 & 0 & $\begin{array}{l}109 \\
(97.3 \%)\end{array}$ & 0 & 0 & $\begin{array}{l}112 \\
(100 \%)\end{array}$ & $\begin{array}{l}19 \\
(16.9 \%)\end{array}$ & $\begin{array}{l}16 \\
(14.3 \%)\end{array}$ & $\begin{array}{l}1 \\
(0.9 \%)\end{array}$ & $\begin{array}{l}95 \\
(84.8 \%)\end{array}$ & 0 & 0 \\
\hline
\end{tabular}




\begin{tabular}{|llllll|}
\hline Year & No. of tested & \multicolumn{2}{c}{ pfmdr1 CNV } & \multicolumn{3}{c|}{ pfpm2 CNV } \\
\cline { 3 - 6 } & & single copy & multiple copy $(>1.5)$ & single copy & multiple copy $(>1.6)$ \\
\hline 2013 & 27 & $24(88.9 \%)$ & $3(11.1 \%)$ & $25(92.6 \%)$ & $2(7.4 \%)$ \\
\hline 2014 & 39 & $37(94.8 \%)$ & $2(5.2 \%)$ & $19(48.7 \%)$ & $20(51.3 \%)$ \\
\hline 2015 & 46 & $46(100 \%)$ & 0 & $10(21.7 \%)$ & $36(78.3 \%)$ \\
\hline Total & 112 & $107(95.5 \%)$ & $5(4.5 \%)$ & $54(48.2 \%)$ & $58(51.8)$ \\
\hline
\end{tabular}

Fig. 1 shows the prevalence of pfpm2 copy number variation, pfmdr1 copy number variation, pfcrt-F145l, pfk13-C580Y and pfk13R539T mutations over time. The prevalence of parasites with multiple pfpm 2 copies associated with PPQ resistance increased from 2013 to 2015, similar to the prevalence of parasites harboring pfcrt-F145I and pfk13-C580Y mutations. In contrast, the prevalence of parasites with multiple pfmdr1 copies associated with MQ resistance and pfk13-R539T mutation decreased after 2013 through 2015.

Table 3. In vitro susceptibility of $P$. falciparum isolates to dihydroartemisinin (DHA), artesunate (AS), artemisone (ATM), chloroquine $(C Q)$, piperaquine $(P P Q)$, cycloguanil (CYC), mefloquine (MQ), quinine (QN), lumefantrine (LUM), doxycycline (DOX), atovaquone (ATQ).

\begin{tabular}{|c|c|c|c|c|c|c|c|c|}
\hline \multirow[b]{2}{*}{ Drug } & \multicolumn{7}{|c|}{ Geometric Mean IC ${ }_{50}(\mathrm{nM})(95 \% \mathrm{Cl})$} & \multirow[t]{2}{*}{$P$ value } \\
\hline & W2 clone & $\mathbf{N}$ & 2013 & $\mathbf{N}$ & 2014 & $\mathbf{N}$ & 2015 & \\
\hline DHA & $3.8(2.9-4.8)$ & 11 & $10.8(8.8-13)$ & 11 & $7.2(5.8-8.9)$ & 18 & $7.1(5.4-9.5)$ & 0.057 \\
\hline AS & $4.8(3.7-6.1)$ & 11 & $8.2(6.7-10)$ & 11 & $4.8(3.9-5.9)$ & 18 & $4.9(3.8-6.2)$ & $0.005^{1,2}$ \\
\hline ATM & $1.5(0.8-2.9)$ & 11 & $1.9(1.1-3.1)$ & 11 & $0.7(0.5-1.0)$ & 18 & $0.9(0.6-1.6)$ & $0.021^{1}$ \\
\hline $\mathrm{CQ}$ & 234 (189-289) & 11 & $89.3(42-190)$ & 11 & $157.4(101-244)$ & 18 & $228.4(145-359)$ & $0.025^{2}$ \\
\hline PPQ & $63(50-78)$ & 11 & $58.5(43-79)$ & 10 & $75.1(54-104)$ & 16 & $406.9(137-1206)$ & $0.006^{2}$ \\
\hline CYC & $\begin{array}{l}3,314(1,945- \\
5,648)\end{array}$ & 8 & 1399 (855-2290) & 6 & $1566(790-3102)$ & 18 & $8642(5086-14684)$ & $0.0008^{2,3}$ \\
\hline MQ & $62.1(52-74)$ & 11 & $18.3(9.9-34)$ & 11 & $35.9(20-63)$ & 18 & $67.4(48.9-93.0)$ & $0.0027^{2}$ \\
\hline QN & $277(232-331)$ & 11 & $68.2(42-110)$ & 11 & 136.8 (99-190) & 18 & $144.9(113-185)$ & $0.023^{2}$ \\
\hline LUM & $3.6(2.4-5.4)$ & 11 & $3.4(2.3-5.0)$ & 11 & $6.5(4.4-9.8)$ & 18 & $4.7(3.5-6.4)$ & 0.052 \\
\hline DOX & $\begin{array}{l}7,580(5,858- \\
9,808)\end{array}$ & 11 & $\begin{array}{l}23092 \text { (19205- } \\
27767)\end{array}$ & 11 & $\begin{array}{l}18491(15206- \\
22485)\end{array}$ & 18 & $\begin{array}{l}14008 \text { (10737- } \\
18276)\end{array}$ & $0.025^{2}$ \\
\hline ATQ & $12.5(7.8-20)$ & 10 & $1.7(0.9-3.2)$ & 11 & $2.5(1.6-3.8)$ & 17 & $3.3(1.8-6.0)$ & 0.253 \\
\hline
\end{tabular}

$P$ values calculated by the Kruskal-Wallis test

1, 2,3 Significant difference calculated by Dunn's multiple comparisons test of data in 2013/2014, 2013/2015, and 2014/2015, respectively

\section{In vitro drug susceptibility of $P$. falciparum isolates.}

Of 86 P. falciparum monoinfection samples that met the parasitemia level criteria for in vitro culture, in vitro drug susceptibility of only 40 samples (11, 11 and 18 samples from 2013, 2014, and 2015, respectively) against antimalarial drug panel could be obtained by HRP2-ELISA. (Table 3). In viewing the geometric mean (GM) IC 50 data for all drugs by year, CQ, PPQ, CYC, MQ, and QN all exhibited statistically significant decreases in drug susceptibility. Compared to the ART- and MQ-sensitive W2 reference clone, isolates had greatly reduced sensitivity to PPQ, CYC, and DOX, with PPQ and CYC having a noticeable drop in effectiveness between 2014 and 2015. In contrast, while there was a steady decline in $C Q$ and $M Q$ sensitivity from 2013 to 2015 but GM-IC $C_{50}$ values remained lower than that of CQ-resistance W2 reference $\left(\mathrm{IC}_{50}-\mathrm{CQ}=234 \mathrm{nM}\right)$ and MQ-resistance $\mathrm{D} 6$ reference $\left(\mathrm{IC}_{50}-\mathrm{MQ}=118 \mathrm{nM}\right)$, respectively. There 
was a modest increase in DHA and AS susceptibility, which did not change dramatically over the 3-year period. All parasite isolates had the IC $\mathrm{I}_{50}$-ATQ lower than that of the ATQ-resistance C2B reference $\left(\mathrm{IC}_{50}-\mathrm{ATQ}=13,240 \mathrm{nM}\right)$, suggesting no ATQ-resistant phenotype. No significant changes in drug susceptibility of DHA, LUM and ATQ were observed during the period of this study.

\section{In vitro Ring stage Survival Assay (RSA) and pfk13 mutations}

To get a better understanding of ART-resistance, 40 isolates from 2013 to 2015 were tested in in vitro RSA $\mathrm{A}_{0-3 \mathrm{~h}}$ to measure $\%$ survival rate against DHA, and the association with pfk13 mutations assessed (Fig. 2A). One isolate was excluded due to the growth rate between 0 and 72 hours being less than 1.5, leaving 39 isolates evaluable by RSA $\mathrm{A}_{0-3 \mathrm{~h}}$. Survival rates for the 11 evaluable isolates from 2013 ranged from 0.84 to $44.5 \%$ (median 19.9, IQR of 3.4-30.3), for the 11 evaluable 2014 isolates the range was 3.7 to $82.8 \%$ (median 16.1, IQR of $9.9-60.9$ ) and for the 17 evaluable isolates in 2015 ranged from 1.4 to $22.8 \% \mathrm{w}$ (median 8.3, IQR of 2.8 -22.8), whereas reference clone survival rate was as expected with medians of $0.4,2.5$, and $23.2 \%$ for ART-sensitive W2, ART-resistant IPC-4884 and IPC-5202, respectively. In 2013, 90.9\% (10/11) were deemed ART-resistant, all of which contained either pfk13-R539T or -C580Y mutations, while the parasite harboring pfk13-R539I was sensitive to ART. In 2014 and 2015, all tested parasites containing either pfk13-R539T or -C580Y mutation were deemed ART resistant. No significant difference in the proportion of resistant isolates between 2013 and 2014 were found $(P>0.05)$ but there was a significant decrease in median survival rate for 2015 (8.3), compared to those from 2014 (16.1) and 2013 (20.9), Mann-Whitney test, $P=0.004$ and $P=0.02$, respectively. It was noted that \% survive rate of the parasite harboring pfk13-R539I was $0.84 \%$, closer to the cut-off value.

\section{PPQ-susceptibility of parasites with PPQ molecular markers}

With the emergence of PPQ resistance associated with DHA-PPQ treatment failures identified in Cambodia from 2010-2013 ${ }^{41}$, the distribution of $\mathrm{PPQ}-\mathrm{IC}_{90}$ values for parasites from Thailand (with newly emerged pfcrt-F145I mutation with or without amplified pfpm2) is displayed in Fig. 2B. It was noted that PPQ-IC g0 $_{90}$ results were evaluable in 11 isolates in 2013, 10 isolates in 2014, and 18 isolates in 2015. In 2013, there was only one isolate with multiple pfpm 2 copies and it had an elevated $I_{9_{0}}$ value. In 2014 , no significant differences in $\mathrm{IC}_{90}$ values in the isolates with single and multiple pfpm 2 copies were observed $(P>0.05)$. In 2015 there was a significant difference in parasites with single (226 nM) and multiple pfpm2 copies $(62,347 \mathrm{nM})$ without pfcrt-F145I $(\mathrm{P}=0.036)$ whereas no significant differences in $\mathrm{IC}_{90}$ values in parasites with single $(53,930 \mathrm{nM})$ and multiple pfpm2 copies $(77,882 \mathrm{nM})$ with pfcrt-F145I $(\mathrm{P}>0.05)$. Isolates with single pfpm2 and wild type pfcrt had more than 10 -fold lower $\mathrm{IC}_{90} \mathrm{~s}$ than parasites with multiple pfpm 2 and/or pfcrt $F 145 I$. With the exception of 1 isolate, parasites harboring $p f c r t F 145 I$ mutation exhibited higher $I_{90}$ values than those without the mutation (PPQ-IC $\mathrm{C}_{90}$ s for standard clone W2, D6 and C2B: 124, 157, and $232 \mathrm{nM}$, respectively).

In the light of an association between PPQ resistance and haplotypes, Table S4 shows PPQ-IC ${ }_{90} \mathrm{~S}$ of the parasite isolates in different haplotype subgroups. It can be clearly seen that parasite isolates containing multiple pfpm2 copies alone (group III), the pfcrt-F145I alone (group V), or the combination of both markers exhibited noticeably high $I_{9_{00}}$ values, indicative of PPQ resistance phenotype.

\section{Preexisting antimalarial treatment}

During the enrollment process, participants were queried about recent malaria infection or antimalarial drug use and also past medical history of malaria. Of 112 participants, 27 (24.1\%) had reported prior malaria infection: 15 (55.6\%), 7 (25.9\%), 3 (11.1\%), and 2 (7.4) had $1,2,3$, and 4 episodes of malaria in the last 12 months. Thirty participants (26.8\%) admitted that they had previously taken antimalarials more than 1 week. The most taken antimalarials was PQ (26.8\%), followed by AS and MQ (16.1\%) and CQ (10.7\%). 18 participants (16.1\%) had taken AS, MQ, and PQ, while 12 participants $(10.7 \%)$ had taken $P Q$ and $C Q$.

Many antimalarials are long-acting or participants may not disclose recent receipt of antimalarial drug; therefore antimalarial drug levels in plasma samples were determined by two independent techniques, ex vivo bioassay and LC-MS/MS, to assess potential effects on study results. In the ex vivo bioassay, 12 of 112 patient plasma samples (10.7\%) evaluated against $P$. falciparum W2 clone had significant antimalarial activity (>17.6 nM DHA equivalents) (Table 4), while $89.3 \%$ of evaluable samples were considered negative $(\leq 17.6 \mathrm{nM})$. The positive results were measured in DHA activity equivalents ranging from 49.8 to $3,855 \mathrm{nM}$ with a median activity of $234.2 \mathrm{nM}$ (IQR of 94.3 to $751.4 \mathrm{nM}$ ). When LC-MS/MS was employed to detect antimalarial drugs in patient plasma samples, 21 samples (18.8\%) were found to contain some antimalarial drug (Table 4). Sixteen of the 21 had MQ, first-line therapy for $P$. falciparum at the time of the study and with a half-life of 8 to 20 days. Six had PQ or CPQ; in 2013-2015 a single dose of PQ had not yet been widely used for $P$. falciparum anti-gametocyte activity but it was first-line for $P$. vivax infections. Interestingly four people had 
evidence of DHA or AS which has a very short half-life (2-3 hours). When comparing both methods, 12 patient plasma samples demonstrated positivity for antimalarial drugs in both ex vivo bioassay and LC-MS/MS method while another 9 samples showed negative result in ex vivo bioassay but were positively detected under LC-MS/MS, in which 3 and 6 samples contained PQ and MQ, respectively. It was important to note that only 8 samples (38.1\%) from the positive samples could be in vitro cultured and evaluated for their drug susceptibility, which showed no significant difference to those without the baseline medicines.

Table 4. Comparison preexisting antimalarial activity in the sample obtained by ex vivo bioassay and liquid chromatography-mass spectrometry (LC-MS/MS).

\begin{tabular}{|lll|}
\hline Sample ID & $\begin{array}{l}\text { Ex vivo Bioassay } \\
\text { (nM DHA equivalents) }\end{array}$ & $\begin{array}{l}\text { Drugs detected } \\
\text { by LC-MS }\end{array}$ \\
\hline BA-005 & 79.4 & MQ, cMQ \\
\hline BA-006 & 90.6 & MQ, cMQ \\
\hline BA-028 & 105.6 & MQ, cMQ \\
\hline BA-032 & - & MQ, cMQ \\
\hline BA-046 & 3,855 & DHA, MQ \\
\hline BA-047 & - & $\mathrm{PQ}, \mathrm{cPQ}$ \\
\hline BA-048 & 207.5 & $\mathrm{MQ}, \mathrm{CMQ}$ \\
\hline BA-053 & - & $\mathrm{PQ}, \mathrm{cPQ}$ \\
\hline BA-055 & - & $\mathrm{MQ}, \mathrm{CMQ}$ \\
\hline BA-057 & 49.8 & $\mathrm{MQ}, \mathrm{cMQ}$ \\
\hline BA-059 & 260.9 & $\mathrm{AS}$ \\
\hline BA-064 & 758.1 & $\mathrm{AS}, \mathrm{DHA}, \mathrm{PQ}, \mathrm{cPQ}$ \\
\hline BA-066 & 731.3 & $\mathrm{AS}, \mathrm{DHA}, \mathrm{MQ}$ \\
\hline BA-068 & - & $\mathrm{MQ}, \mathrm{CMQ}$ \\
\hline BA-072 & - & $\mathrm{PQ}, \mathrm{CPQ}$ \\
\hline BA-078 & 191.4 & $\mathrm{MQ}, \mathrm{cMQ}$ \\
\hline BA-084 & 840.4 & $\mathrm{DHA}, \mathrm{PQ}, \mathrm{CPQ}, \mathrm{MQ}, \mathrm{cMQ}$ \\
\hline BA-087 & 468.4 & $\mathrm{MQ}, \mathrm{cPQ}, \mathrm{MQ}, \mathrm{cMQ}$ \\
\hline BA-095 & - & $\mathrm{MQ}, \mathrm{CMQ}$ \\
\hline BA-103 & - & $\mathrm{MQ}, \mathrm{CMQ}$ \\
\hline BA-110 & - & \\
\hline & & \\
\hline
\end{tabular}

\section{Discussion}

Even though the Greater Mekong Subregion (GMS) has long been the epicentre of antimalarial drug resistance, these countries are aiming to achieve malaria elimination by $2030^{50}$. At the time this study was enrolling volunteers, the only validated molecular markers for ACTs were pfmdr1 but by time of writing this manuscript we developed assays to delineate markers and in vitro assays to ART, $\mathrm{PPQ}$, and ATQ resistance. As the number of malaria cases in the GMS continues to decline following aggressive malaria control efforts, conducting $P$. falciparum therapeutic efficacy studies has become more difficult. Therefore, current efforts have to rely more on the surveillance of molecular markers in concert with in vitro data. This type of intensive surveillance of molecular and in vitro data with a minimum of 6 week patient follow up is effective and easily adapted to mobile populations such as military were the majority of volunteers enrolled. Similar approaches can be applied to migrants and occupations where follow up post treatment is difficult, 
including workers in forestry, agriculture or animal husbandry and refugee populations. Despite the limitations of the study, where clinical treatment outcomes were not readily available, we were able to characterize the changes in drug resistance overtime in this difficult to reach population. Since most cases of malaria in Thailand occur in border regions, areas where a military presence is required, Thai soldiers stationed at these locations are at increased risk of developing multidrug resistant malaria. There has been limited information published on drug resistance in the military cohorts and this provides new insights.

Chloroquine resistance was first reported in the GMS since the $1950 \mathrm{~s}^{51}$, and several groups ${ }^{52-56}$ have characterized CQ resistant parasite lineages as one of four mutant pfcrt genotypes at positions 72-76 (CVIET, SVMNI, CVMNI, and CVMET; mutation underlined), with the SVMNI genotype being detected in Brazil/Peru and Melanesia lineages ${ }^{57,58}$. Mutations in pfcrt are associated with CQ, AQ and LUM resistance; especially, the pfcrt 72-76 CVIET and SVMNT haplotype. The 76T point mutation is the key marker, while SVMNT haplotype is required for $A Q$ resistance ${ }^{53,55}$. The present results show that all collected parasite isolates harbored the pfcrt 72-76 CVIET haplotype similar to previous reports ${ }^{59-61}$. Even though CQ sensitivity of the parasite isolates was still slightly higher than that of the CQ-resistance W2 reference clone, when the geometric mean $I_{50} S$ of the CQ-sensitive isolates of $30.1 \mathrm{nM}^{62}$ was applied, all the parasites collected under this study would be deemed CQ-resistant. Decreased CQ sensitivity was observed from 2013 to 2015 that can likely be attributed to continued CQ use in Thailand for treatment of $P$. vivax.

Parasite isolates carrying the artemisinin resistant gene have been reported in Thailand ${ }^{61,63}$. Some pfk13 mutations (F446I, N458Y, M476I, Y493H, R539T, I543T, P553L, R561H and C580Y) were validated for conferring artemisinin resistance ${ }^{64}$, while several other mutations may be deemed candidates. The current study showed that the pfk13-C580Y mutation was predominant, rising from $63 \%$ in 2013 to $100 \%$ in 2015 . These findings are in good agreement with those reported by others ${ }^{42,61}$ in that several pfk 13 mutations developed in Sisaket $(62 \% 580 Y)^{42}$ but by 2015 the C580Y mutation became the sole predominant mutation found ${ }^{61}$. This pattern was not followed by other regions of Thailand. In southern Thailand (Yala Province) very few isolates with $p f k 13$ mutations have been detected $^{61,65}$. The pfk13-R539T has been previously identified in Thailand, Cambodia, Laos, and Vietnam ${ }^{66-69}$; however, the pfk13R539l found in this study has not yet been reported in Thailand. The pfk 13-R539I mutation was previously observed at $0.9 \%$ in Ghanaian isolates ${ }^{70}$. As assessed by the RSA $A_{0-3 h}$, the R539I mutation appears not to be associated with ART resistance. The R539I mutation provided the lower stabilization score, implying an effect on the protein structure and function ${ }^{70}$. All the collected isolates also had a wild-type cytochrome b gene (pfcytb) at both codons 258 and 268 (those associated with atovaquone/proguanil treatment failure ${ }^{36,38,71}$ ) which is consistent with other isolates collected along Thai-Myanmar and Thai-Cambodia borders during 1998-2005 ${ }^{72}$. Additionally, no noticeable increase in atovaquone $\mathrm{IC}_{50}$ of parasites from this study was observed in comparison to the previous report, suggesting that atovaquone/proguanil can still be utilized for treatment and prophylaxis of multidrug-resistant $P$. falciparum malaria in Thailand. Of all the pfmdr1 SNPs analyzed, allelic variation was only observed in $p f m d r 1$ position 184 . This is in good agreement with the previous report by Thita et al. ${ }^{73}$, in which $89 \%$ of $P$. falciparum isolates from Thai-Cambodia border from Chanthaburi and Trat province from 1988 to 2016 had the pfmdr1 184F allele. Previous studies in Uganda and Bioko Island suggest that this allele may play a certain role in mediating resistance to some antimalarials ${ }^{74,75}$, and while in the current study, decreased susceptibility to QN, CQ, and MQ was observed, causality cannot be established. Different results were observed from the $P$. falciparum isolates from the southern part of Thailand, with the pfmdr1 86Y allele significantly more common ${ }^{76}$.

In addition to SNPs in established malaria resistance markers, pfpm2 and pfmdr1 copy numbers were quantified as associated with $\mathrm{PPQ}$ and $\mathrm{MQ}$ resistance, respectively. The increased trend in multiple $p f p m 2$ copy numbers were clearly seen with a decline in multiple pfmdr1 copy numbers. Novel mutations of the pfcrt gene downstream of the 4-aminoquinoline resistance locus (positions 72 to 76 ), including T93S, H97Y, F145I, I218F, M343L, or G353V, were recently shown to be associated with PPQ resistance $30,32,33$. From this study, there was a relatively rapid rise in the $\mathrm{F} 145 \mathrm{I}$ mutation, from $0 \%$ in 2013 to $30 \%$ in 2015 . It cannot be ruled out that other novel pfcrt mutations may have been present in the RTA samples presented here, since they were not studied. In this study, $I_{90}$ of $P P Q$ were employed to investigate the PPQ resistant phenotypes associated with the tested genetic markers. Parasite isolates holding either multiple pfpm 2 copies with pfcrt-F145 or the combination of single or multiple pfpm 2 copy numbers with pfcrt-F145I mutation exhibited the elevated $\mathrm{IC}_{90}$ values of PPQ. Some parasite isolates harboring multiple pfpm2 copy numbers without $p f c r t-F 145 \mathrm{I}$ mutation seemed to have a broad IC $\mathrm{I}_{90}$ values (203 to $5,376 \mathrm{nM}$ ), while parasite isolates with a single $p f p m 2$ copy number had the $\mathrm{IC}_{90}$ values around 71 to $1,183 \mathrm{nM}$. The high $\mathrm{IC}_{90}$ values of parasite isolates with only multiple $p f p m 2$ copy numbers could be stemmed from other novel pfcrt mutations that were not detected in this study. Previous studies have shown that the overexpression of $p f p m 2$ and pfpm3 in the 3D7 genetic background did not alter the sensitivity of $P$. falciparum to PPQ, suggesting that the increase in $p f p m 2$

Page $11 / 19$ 
copy number alone is not the sole modulator of PPQ resistance 47,77 , the initial selection of pfpm2 and pfmdr1 copy number variations developed a genetic background important for novel pfcrt mutations to emerge ${ }^{78}$. The increased pfpm2 copy numbers was also accompanied with the escalated numbers of pfk13-C580Y and pfcrt-F145I mutations.

Overall, from 2013-2015, P. falciparum isolates collected from the military population demonstrated progression from $62 \%$ to $100 \%$ $580 Y$ pfk 13 mutations, all with increased survival rates by RSA. Regarding ACT partner drugs, there was a decrease in multiple $p f m d r 1$ copy numbers from $11 \%$ to $0 \%$ and a corresponding increase of multiple pfpm 2 copy numbers or multiple pfpm 2 copy numbers with pfcrt-F145I mutation from $7.4 \%$ to $78.3 \%$. The first-line antimalarial used during this time was AS-MQ, so the drive toward increasing PPQ resistance likely came from cross-border mixing of parasite populations from Cambodia. In 2013, an AFRIMS study in Anlong Veng, Cambodia, $12 \mathrm{~km}$ from the Cambodia-Thai border at Sisaket province, showed a similar rate of 65\% C580Y pfk 13 mutant ${ }^{41}$. When the $54 \%$ failure rate of DHA-PPQ was observed in Anlong Veng in $2013^{41}$, the molecular markers for neither ART nor PPQ were available so it is only retrospectively that the association of molecular markers and in vitro data can be seen. Similarly in Sisaket, the 87\% failure rate of DHA-PPQ from the TRACII study (2015-2018) ${ }^{63}$ may have been predicted from these surveillance data.

The benefits of having information about past medical history of malaria can help interpret ex vivo/in vitro drug testing results obtained from study samples. It also helps national programs better understand how often anti-malarial drugs are being used within communities and if this use is outside regulated dispensing of drugs by health care providers. The use of previous antimalarial drugs or preexisting antimalarial activity in patients can have an effect on malaria treatment ${ }^{46}$. A bioassay method was developed for the measurement of antimalarial activities of ART and its derivatives in either the plasma or sera of patients ${ }^{79,80}$, while bioanalytical LCMS/MS has widely been used to detect several drugs ${ }^{81,82}$. In this study, results from bioassay demonstrated a good correlation to LCMS/MS determination for blood stage antimalarial activities. The factors that limit the detection of the two techniques may lie on the drug metabolites and the amount of the drugs in the samples. For example, 3 samples found positively in LC-MS/MS method contained PQ, the erythrocytic activity of which is little, hence not detected in ex vivo bioassay. Another 6 samples, on the other hand, were found negative in ex vivo bioassay but positive in LC-MS/MS method with the presence of MQ and CMQ at the concentration lower than $150 \mathrm{ng} / \mathrm{ml}$. These results suggested that bioassay was able to screen blood stage antimalarial activity in certain levels but could not specify which drugs or metabolites are present in the patient plasma samples whereas LC-MS/MS method could. Therefore, it will be fruitful to develop LC-MS/MS method to simultaneously screen multiple antimalarial drugs and their metabolites in a single run.

The difficulty of choosing a national first-line therapy in the GMS due to differing patterns of antimalarial resistance as illustrated. High rates of AS-MQ failures along the Thai-Myanmar border ${ }^{16,83}$ resulted in the first-line treatment being changed to DHA-PPQ in 2015. However, the parasites from this high risk mobile military population had patterns developed over three years which were more similar to isolates in Cambodia, and then manifested a similar drop in DHA-PPQ efficacy. The Thai government responded to that regional challenge and since 2019, the first-line treatment for $P$. falciparum has been changed to artesunate-pyronaridine (AS-PND) in Sisaket and Ubon Ratchathani Provinces. Recent reports suggest there are two lineages within the GMS. For pfk 13 genotypes, the C580Y mutation predominates in the eastern GMS and the F466l mutation has spread along the Myanmar border ${ }^{61}$. Similarly, pfpm2 gene amplification has been found in northeastern Thailand near Cambodia border, but not in western and southern Thailand ${ }^{61}$. It is interesting to note that $P$. falciparum isolates from eastern Greater Mekong has different mechanism of pfpm2 amplification from those in Myanmar as judged by the sequences flanking the amplified region ${ }^{61}$. While there may be predominant lineages which have emerged, continued local surveillance will be important to determine if and how these patterns shift. The emergence of the novel pfcrt F145I in 2014 from this study followed the emergence and DHA-PPQ treatment failures in Cambodia ${ }^{41,46,84}$. Shrestha, et al. ${ }^{85}$ showed that more than $98 \%$ of the parasites collected from northern Cambodia carried newly emerged pfcrt mutations and after 2014, the prevalence of parasites with pfcrt-F145I mutation started to decrease, being out-competed by other less resistant, but more fit pfcrt variants such as pfcrt-T93S and $1218 \mathrm{~F}^{33}$. Recently, Imwong, et al. ${ }^{61}$ has reported that pfcrt-G353V was detected in 7 of 142 isolates and 7 of 38 isolates after mass drug administration with DHA-PPQ in Cambodia. Understanding the movement of human populations especially along border areas in migrants and militaries, with ongoing surveillance programs can help predict drug efficacy or first detect any introduction of novel mutations which may interrupt the path to malaria elimination in the GMS.

\section{Conclusion}


Altogether, data from the molecular, in vitro drug susceptibility and survival assay indicate the increased trend of $p f k 13-\mathrm{C} 580 \mathrm{Y}$ mutation, multiple $p f p m 2$ copy numbers and $p f c r t F 145 \mathrm{I}$ which is indicative of ART and PPQ resistance of Thai $P$. falciparum isolates collected near the Cambodia border, at the same time when DHA-PPQ treatment failures in Cambodia were on the rise. The sharp rise of $\mathrm{IC}_{90}$ values was found to be linked to the presence of multiple pfpm 2 copies and pfcrt $\mathrm{F} 145 \mathrm{I}$ mutation, whereas the $p f k 13-\mathrm{R} 539 \mathrm{I}$ mutation was not associated with ART resistance according to RSA. The observed drop in PPQ, MQ, and CYC sensitivity could be an alarming issue for areas where treatment options remain limited.

\section{Declarations}

\section{Acknowledgements}

The following reagents were obtained through BEI Resources, NIAID, NIH: Plasmodium falicaprum, Strain IPC-4884 (MRA-1238) and IPC-5202 (MRA-1240), contributed by Didier Ménard. The authors would like to thank the clinical and laboratory staff who made the study possible. The views expressed in this article are those of the authors and do not reflect the official policy of the Department of the Army, Department of Defense, U.S. or Thai Governments.

\section{Author's contributions}

DLS, KJ and JG conceived, designed and executed the study. NB, PV, PL, PG, DU, WR, CL, DW, NS, SC2, WK, BAV, MDS, MMF, DLS, PLS, MW, and NCW conceived, designed and supported the study. NU and MA were in charge of sample collection and transportation. CT, SS, SC1, CP, WF performed cell culture, drug susceptibility assay, survival assay and bioassay. KK, CC and PS performed molecular genotyping. WT performed LC-MS/MS. NB and PV initially analyzed the data and all authors assisted in interpreting the data. NB wrote the first draft. All authors edited, reviewed and approved the final manuscript.

\section{Disclaimer}

Material has been reviewed by the Walter Reed Army Institute of Research. There is no objection to its presentation/publication. The opinions or assertions contained herein are the private views of the author, and are not to be construed as official, or as reflecting true views of the Department of the Army or the Department of Defense. The investigators have adhered to the policies for protection of human subjects as prescribed in AR 70-25.

\section{Ethics approval and consent to participate}

All participants or guardians provided written consent and samples were collected under approval Royal Thai Army Institutional Review Board (RTA IRB) and Walter Reed Army Institute of Research Institutional Review board.

\section{Consent for publication}

Not applicable

\section{Availability of data and materials}

All data generated or analyzed during this study are included in this published article and its supplementary information files.

\section{Competing Interests}

The authors declare that they have no competing interests.

\section{Funding}

This work was supported by the United States Department of Defense Armed Forces Health Surveillance Division-Global Emerging Infectious Disease Surveillance Branch (AFHSD-GEIS), Funding site No. IM084 and Defense Malaria Assistance Program (DMAP). The funding source had no role in the analysis or interpretation of data, preparation of the manuscript or the decision to publish.

\section{References}

1. World Malaria Report. (World Health Organization, Geneva, 2020). 
2. Luxemburger, C. et al. Two patients with falciparum malaria and poor in vivo responses to artesunate. Transactions of the Royal Society of Tropical Medicine and Hygiene. 92, 668-669 https://doi.org/10.1016/s0035-9203(98)90807-0 (1998).

3. Wongsrichanalai, C. \& Meshnick, S. R. Declining artesunate-mefloquine efficacy against falciparum malaria on the CambodiaThailand border. Emerg Infect Dis. 14, 716-719 https://doi.org/10.3201/eid1405.071601 (2008).

4. Noedl, H. et al. Evidence of artemisinin-resistant malaria in western Cambodia. The New England journal of medicine. 359, 26192620 https://doi.org/10.1056/NEJMc0805011 (2008).

5. Dondorp, A. M. et al. Artemisinin resistance in Plasmodium falciparum malaria. The New England journal of medicine. 361, 455467 https://doi.org/10.1056/NEJMoa0808859 (2009).

6. Amaratunga, C. et al. Artemisinin-resistant Plasmodium falciparum in Pursat province, western Cambodia: a parasite clearance rate study. The Lancet. Infectious diseases. 12, 851-858 https://doi.org/10.1016/S1473-3099(12)70181-0 (2012).

7. Phyo, A. P. et al. Emergence of artemisinin-resistant malaria on the western border of Thailand: a longitudinal study. Lancet. 379, 1960-1966 https://doi.org/10.1016/S0140-6736(12)60484-X (2012).

8. Kyaw, M. P. et al. Reduced susceptibility of Plasmodium falciparum to artesunate in southern Myanmar. PLoS One. 8, e57689 https://doi.org/10.1371/journal.pone.0057689 (2013).

9. Ashley, E. A. et al. Spread of artemisinin resistance in Plasmodium falciparum malaria. The New England journal of medicine. 371, 411-423 https://doi.org/10.1056/NEJMoa1314981 (2014).

10. Ariey, F. et al. A molecular marker of artemisinin-resistant Plasmodium falciparum malaria. Nature. 505, 50-55 https://doi.org/10.1038/nature12876 (2014).

11. Ghorbal, M. et al. Genome editing in the human malaria parasite Plasmodium falciparum using the CRISPR-Cas9 system. Nature biotechnology. 32, 819-821 https://doi.org/10.1038/nbt.2925 (2014).

12. Fairhurst, R. M. Understanding artemisinin-resistant malaria: what a difference a year makes. Current opinion in infectious diseases. 28, 417-425 https://doi.org/10.1097/QC0.0000000000000199 (2015).

13. Straimer, J. et al. Drug resistance. K13-propeller mutations confer artemisinin resistance in Plasmodium falciparum clinical isolates. Science. 347, 428-431 https://doi.org/10.1126/science.1260867 (2015).

14. Witkowski, B. et al. Novel phenotypic assays for the detection of artemisinin-resistant Plasmodium falciparum malaria in Cambodia: in-vitro and ex-vivo drug-response studies. The Lancet. Infectious diseases. 13, 1043-1049 https://doi.org/10.1016/S1473-3099(13)70252-4 (2013).

15. Price, R. N. et al. Mefloquine resistance in Plasmodium falciparum and increased pfmdr1 gene copy number. Lancet. 364, 438447 https://doi.org/10.1016/S0140-6736(04)16767-6 (2004).

16. Phyo, A. P. et al. Declining Efficacy of Artemisinin Combination Therapy Against P. Falciparum Malaria on the Thai-Myanmar Border (2003-2013): The Role of Parasite Genetic Factors. Clinical infectious diseases: an official publication of the Infectious Diseases Society of America. 63, 784-791 https://doi.org/10.1093/cid/ciw388 (2016).

17. Eyase, F. L. et al. The role of Pfmdr1 and Pfcrt in changing chloroquine, amodiaquine, mefloquine and lumefantrine susceptibility in western-Kenya P. falciparum samples during 2008-2011. PLoS One. 8, e64299 https://doi.org/10.1371/journal.pone.0064299 (2013).

18. Foote, S. J. et al. Several alleles of the multidrug-resistance gene are closely linked to chloroquine resistance in Plasmodium falciparum. Nature. 345, 255-258 https://doi.org/10.1038/345255a0 (1990).

19. Povoa, M. M. et al. Pfmdr1 Asn1042Asp and Asp1246Tyr polymorphisms, thought to be associated with chloroquine resistance, are present in chloroquine-resistant and -sensitive Brazilian field isolates of Plasmodium falciparum. Exp Parasitol. 88, 64-68 https://doi.org/10.1006/expr.1998.4195 (1998).

20. Sidhu, A. B., Valderramos, S. G. \& Fidock, D. A. pfmdr1 mutations contribute to quinine resistance and enhance mefloquine and artemisinin sensitivity in Plasmodium falciparum. Molecular microbiology. 57, 913-926 https://doi.org/10.1111/j.13652958.2005.04729.x (2005).

21. Preechapornkul, P. et al. Plasmodium falciparum pfmdr1 amplification, mefloquine resistance, and parasite fitness. Antimicrob Agents Chemother. 53, 1509-1515 https://doi.org/10.1128/AAC.00241-08 (2009).

22. Borges, S. et al. Genomewide scan reveals amplification of $\mathrm{mdr} 1$ as a common denominator of resistance to mefloquine, lumefantrine, and artemisinin in Plasmodium chabaudi malaria parasites. Antimicrob Agents Chemother. 55, 4858-4865 https://doi.org/10.1128/AAC.01748-10 (2011).

Page $14 / 19$ 
23. Lekana-Douki, J. B. et al. Increased prevalence of the Plasmodium falciparum Pfmdr1 $86 \mathrm{~N}$ genotype among field isolates from Franceville, Gabon after replacement of chloroquine by artemether-lumefantrine and artesunate-mefloquine. Infect Genet Evol. 11, 512-517 https://doi.org/10.1016/j.meegid.2011.01.003 (2011).

24. Ngo, T. et al. Analysis of pfcrt, pfmdr1, dhfr, and dhps mutations and drug sensitivities in Plasmodium falciparum isolates from patients in Vietnam before and after treatment with artemisinin. The American journal of tropical medicine and hygiene. 68, 350356 (2003).

25. Phompradit, P., Wisedpanichkij, R., Muhamad, P., Chaijaroenkul, W. \& Na-Bangchang, K. Molecular analysis of pfatp6 and pfmdr1 polymorphisms and their association with in vitro sensitivity in Plasmodium falciparum isolates from the Thai-Myanmar border. Acta Trop. 120, 130-135 https://doi.org/10.1016/j.actatropica.2011.07.003 (2011).

26. Amato, R. et al. Genetic markers associated with dihydroartemisinin-piperaquine failure in Plasmodium falciparum malaria in Cambodia: a genotype-phenotype association study. The Lancet. Infectious diseases. 17, 164-173 https://doi.org/10.1016/S1473-3099(16)30409-1 (2017).

27. Parobek, C. M. et al. Partner-Drug Resistance and Population Substructuring of Artemisinin-Resistant Plasmodium falciparum in Cambodia. Genome biology and evolution. 9, 1673-1686 https://doi.org/10.1093/gbe/evx126 (2017).

28. Witkowski, B. et al. A surrogate marker of piperaquine-resistant Plasmodium falciparum malaria: a phenotype-genotype association study. The Lancet. Infectious diseases. 17, 174-183 https://doi.org/10.1016/S1473-3099(16)30415-7 (2017).

29. Bopp, S. et al. Plasmepsin II-III copy number accounts for bimodal piperaquine resistance among Cambodian Plasmodium falciparum. Nat Commun. 9, 1769 https://doi.org/10.1038/s41467-018-04104-z (2018).

30. Duru, V. et al. Plasmodium falciparum dihydroartemisinin-piperaquine failures in Cambodia are associated with mutant K13 parasites presenting high survival rates in novel piperaquine in vitro assays: retrospective and prospective investigations. $B M C$ medicine. 13, 305 https://doi.org/10.1186/s12916-015-0539-5 (2015).

31. Agrawal, S. et al. Association of a Novel Mutation in the Plasmodium falciparum Chloroquine Resistance Transporter With Decreased Piperaquine Sensitivity. The Journal of infectious diseases. 216, 468-476 https://doi.org/10.1093/infdis/jix334 (2017).

32. Ross, L. S. et al. Emerging Southeast Asian PfCRT mutations confer Plasmodium falciparum resistance to the first-line antimalarial piperaquine. Nat Commun. 9, 3314 https://doi.org/10.1038/s41467-018-05652-0 (2018).

33. Dhingra, S. K., Small-Saunders, J. L., Menard, D. \& Fidock, D. A. Plasmodium falciparum resistance to piperaquine driven by PfCRT. The Lancet. Infectious diseases. 19, 1168-1169 https://doi.org/10.1016/S1473-3099(19)30543-2 (2019).

34. Thanh, N. V. et al. Rapid decline in the susceptibility of Plasmodium falciparum to dihydroartemisinin-piperaquine in the south of Vietnam. Malar J. 16, 27 https://doi.org/10.1186/s12936-017-1680-8 (2017).

35. Chaorattanakawee, S. et al. Ex vivo piperaquine resistance developed rapidly in Plasmodium falciparum isolates in northern Cambodia compared to Thailand. Malar J. 15, 519 https://doi.org/10.1186/s12936-016-1569-y (2016).

36. Korsinczky, M. et al. Mutations in Plasmodium falciparum cytochrome $b$ that are associated with atovaquone resistance are located at a putative drug-binding site. Antimicrob Agents Chemother. 44, 2100-2108 https://doi.org/10.1128/aac.44.8.21002108.2000 (2000).

37. Schwobel, B., Alifrangis, M., Salanti, A. \& Jelinek, T. Different mutation patterns of atovaquone resistance to Plasmodium falciparum in vitro and in vivo: rapid detection of codon 268 polymorphisms in the cytochrome $b$ as potential in vivo resistance marker. Malar J. 2, 5 https://doi.org/10.1186/1475-2875-2-5 (2003).

38. Plucinski, M. M. et al. Novel Mutation in Cytochrome B of Plasmodium falciparum in One of Two Atovaquone-Proguanil Treatment Failures in Travelers Returning From Same Site in Nigeria. Open Forum Infect Dis. 1, ofu059 https://doi.org/10.1093/ofid/ofu059 (2014).

39. Imwong, M., Hien, T. T., Thuy-Nhien, N. T., Dondorp, A. M. \& White, N. J. Spread of a single multidrug resistant malaria parasite lineage (PfPailin) to Vietnam. The Lancet. Infectious diseases. 17, 1022-1023 https://doi.org/10.1016/S1473-3099(17)30524-8 (2017).

40. Imwong, M. et al. The spread of artemisinin-resistant Plasmodium falciparum in the Greater Mekong subregion: a molecular epidemiology observational study. The Lancet. Infectious diseases. 17, 491-497 https://doi.org/10.1016/S1473-3099(17)30048-8 (2017). 
41. Spring, M. D. et al. Dihydroartemisinin-piperaquine failure associated with a triple mutant including kelch 13 C580Y in Cambodia: an observational cohort study. The Lancet. Infectious diseases. 15, 683-691 https://doi.org/10.1016/S1473-3099(15)70049-6 (2015).

42. Talundzic, E. et al. Selection and spread of artemisinin-resistant alleles in Thailand prior to the global artemisinin resistance containment campaign. PLoS pathogens. 11, e1004789 https://doi.org/10.1371/journal.ppat.1004789 (2015).

43. Dow, G. S. et al. Radical curative efficacy of tafenoquine combination regimens in Plasmodium cynomolgi-infected Rhesus monkeys (Macaca mulatta). Malar. J. 10, 212 (2011).

44. Trager, W. \& Jensen, J. B. Human malaria parasites in continuous culture. Science. 193, 673-675 https://doi.org/10.1126/science.781840 (1976).

45. Chaorattanakawee, S. et al. Direct comparison of the histidine-rich protein-2 enzyme-linked immunosorbent assay (HRP-2 ELISA) and malaria SYBR green I fluorescence (MSF) drug sensitivity tests in Plasmodium falciparum reference clones and fresh ex vivo field isolates from Cambodia. Malar J. 12, 239 https://doi.org/10.1186/1475-2875-12-239 (2013).

46. Chaorattanakawee, S. et al. Ex Vivo Drug Susceptibility Testing and Molecular Profiling of Clinical Plasmodium falciparum Isolates from Cambodia from 2008 to 2013 Suggest Emerging Piperaquine Resistance. Antimicrob Agents Chemother. 59, 46314643 https://doi.org/10.1128/AAC.00366-15 (2015).

47. Boonyalai, N. et al. Piperaquine resistant Cambodian Plasmodium falciparum clinical isolates: in vitro genotypic and phenotypic characterization. Malar J. 19, 269 https://doi.org/10.1186/s12936-020-03339-w (2020).

48. Noedl, H., Teja-Isavadharm, P. \& Miller, R. S. Nonisotopic, semiautomated plasmodium falciparum bioassay for measurement of antimalarial drug levels in serum or plasma. Antimicrob Agents Chemother. 48, 4485-4487 https://doi.org/10.1128/AAC.48.11.4485-4487.2004 (2004).

49. Teja-Isavadharm, P. et al. Plasmodium falciparum-based bioassay for measurement of artemisinin derivatives in plasma or serum. Antimicrob Agents Chemother. 48, 954-960 https://doi.org/10.1128/aac.48.3.954-960.2004 (2004).

50. Strategy for malaria elimination in the GMS (2015-2030). (World Health Organization, 2015).

51. Ridley, R. G. Medical need, scientific opportunity and the drive for antimalarial drugs. Nature. 415, 686-693 https://doi.org/10.1038/415686a (2002).

52. Wellems, T. E. et al. Chloroquine resistance not linked to mdr-like genes in a Plasmodium falciparum cross. Nature. 345, 253-255 https://doi.org/10.1038/345253a0 (1990).

53. Wellems, T. E., Walker-Jonah, A. \& Panton, L. J. Genetic mapping of the chloroquine-resistance locus on Plasmodium falciparum chromosome 7. Proc Natl Acad Sci U S A. 88, 3382-3386 https://doi.org/10.1073/pnas.88.8.3382 (1991).

54. Su, X., Kirkman, L. A., Fujioka, H. \& Wellems, T. E. Complex polymorphisms in an approximately $330 \mathrm{kDa}$ protein are linked to chloroquine-resistant P. falciparum in Southeast Asia and Africa. Cell. 91, 593-603 https://doi.org/10.1016/s00928674(00)80447-x (1997).

55. Fidock, D. A. et al. Mutations in the P. falciparum digestive vacuole transmembrane protein PfCRT and evidence for their role in chloroquine resistance. Molecular cell. 6, 861-871 https://doi.org/10.1016/s1097-2765(05)00077-8 (2000).

56. Sidhu, A. B., Verdier-Pinard, D. \& Fidock, D. A. Chloroquine resistance in Plasmodium falciparum malaria parasites conferred by pfcrt mutations. Science. 298, 210-213 https://doi.org/10.1126/science.1074045 (2002).

57. Wootton, J. C. et al. Genetic diversity and chloroquine selective sweeps in Plasmodium falciparum. Nature. 418, 320-323 https://doi.org/10.1038/nature00813 (2002).

58. Chen, N. et al. pfcrt Allelic types with two novel amino acid mutations in chloroquine-resistant Plasmodium falciparum isolates from the Philippines. Antimicrob Agents Chemother. 47, 3500-3505 https://doi.org/10.1128/aac.47.11.3500-3505.2003 (2003).

59. Takahashi, N. et al. Large-scale survey for novel genotypes of Plasmodium falciparum chloroquine-resistance gene pfcrt. Malar J. 11, 92 https://doi.org/10.1186/1475-2875-11-92 (2012).

60. Buppan, P. et al. Multiple Novel Mutations in Plasmodium falciparum Chloroquine Resistance Transporter Gene during Implementation of Artemisinin Combination Therapy in Thailand. The American journal of tropical medicine and hygiene. 99, 987-994 https://doi.org/10.4269/ajtmh.18-0401 (2018).

61. Imwong, M. et al. Molecular epidemiology of resistance to antimalarial drugs in the Greater Mekong subregion: an observational study. The Lancet. Infectious diseases. https://doi.org/10.1016/S1473-3099(20)30228-0 (2020). 
62. Bickii, J., Basco, L. K. \& Ringwald, P. Assessment of three in vitro tests and an in vivo test for chloroquine resistance in Plasmodium falciparum clinical isolates. Journal of clinical microbiology. 36, 243-247 https://doi.org/10.1128/JCM.36.1.243247.1998 (1998).

63. van der Pluijm, R. W. et al. Determinants of dihydroartemisinin-piperaquine treatment failure in Plasmodium falciparum malaria in Cambodia, Thailand, and Vietnam: a prospective clinical, pharmacological, and genetic study. The Lancet. Infectious diseases. 19, 952-961 https://doi.org/10.1016/S1473-3099(19)30391-3 (2019).

64. Artemisinin resistance and artemisinin-based combination therapy efficacy. (World Health Organization, 2018).

65. Noisang, C. et al. Molecular detection of drug resistant malaria in Southern Thailand. Malar J. 18, 275 https://doi.org/10.1186/s12936-019-2903-y (2019).

66. Menard, D. et al. A Worldwide Map of Plasmodium falciparum K13-Propeller Polymorphisms. The New England journal of medicine. 374, 2453-2464 https://doi.org/10.1056/NEJMoa1513137 (2016).

67. Ye, R. et al. Distinctive origin of artemisinin-resistant Plasmodium falciparum on the China-Myanmar border. Sci Rep. 6, 20100 https://doi.org/10.1038/srep20100 (2016).

68. Thuy-Nhien, N. et al. K13 Propeller Mutations in Plasmodium falciparum Populations in Regions of Malaria Endemicity in Vietnam from 2009 to 2016. Antimicrob Agents Chemother. 61, https://doi.org/10.1128/AAC.01578-16 (2017).

69. Iwagami, M. et al. Heterogeneous distribution of k13 mutations in Plasmodium falciparum in Laos. Malar J. 17, 483 https://doi.org/10.1186/s12936-018-2625-6 (2018).

70. Matrevi, S. A. et al. Plasmodium falciparum Kelch Propeller Polymorphisms in Clinical Isolates from Ghana from 2007 to 2016. Antimicrob Agents Chemother. 63, https://doi.org/10.1128/AAC.00802-19 (2019).

71. Fivelman, Q. L., Butcher, G. A., Adagu, I. S., Warhurst, D. C. \& Pasvol, G. Malarone treatment failure and in vitro confirmation of resistance of Plasmodium falciparum isolate from Lagos, Nigeria. Malar J. 1, 1 https://doi.org/10.1186/1475-2875-1-1 (2002).

72. Khositnithikul, R., Tan-Ariya, P. \& Mungthin, M. In vitro atovaquone/proguanil susceptibility and characterization of the cytochrome b gene of Plasmodium falciparum from different endemic regions of Thailand. Malar J. 7, 23 https://doi.org/10.1186/1475-28757-23 (2008).

73. Thita, T. et al. Phenotypic and genotypic characterization of Thai isolates of Plasmodium falciparum after an artemisinin resistance containment project. Malar J. 17, 197 https://doi.org/10.1186/s12936-018-2347-9 (2018).

74. Dokomajilar, C., Nsobya, S. L., Greenhouse, B., Rosenthal, P. J. \& Dorsey, G. Selection of Plasmodium falciparum pfmdr1 alleles following therapy with artemether-lumefantrine in an area of Uganda where malaria is highly endemic. Antimicrob Agents Chemother. 50, 1893-1895 https://doi.org/10.1128/AAC.50.5.1893-1895.2006 (2006).

75. Li, J. et al. High prevalence of pfmdr1 N86Y and Y184F mutations in Plasmodium falciparum isolates from Bioko Island, Equatorial Guinea. Pathogens and global health. 108, 339-343 https://doi.org/10.1179/2047773214Y.0000000158 (2014).

76. Mungthin, M. et al. Distribution of pfmdr1 polymorphisms in Plasmodium falciparum isolated from Southern Thailand. Malar J. 13, 117 https://doi.org/10.1186/1475-2875-13-117 (2014).

77. Loesbanluechai, D. et al. Overexpression of plasmepsin II and plasmepsin III does not directly cause reduction in Plasmodium falciparum sensitivity to artesunate, chloroquine and piperaquine. Int J Parasitol Drugs Drug Resist. 9, 16-22 https://doi.org/10.1016/j.ijpddr.2018.11.004 (2018).

78. Silva, M., Calcada, C., Teixeira, M., Veiga, M. I. \& Ferreira, P. E. Multigenic architecture of piperaquine resistance trait in Plasmodium falciparum. The Lancet. Infectious diseases. 20, 26-27 https://doi.org/10.1016/S1473-3099(19)30689-9 (2020).

79. Traore, B., Lazaro, E. \& Gay, F. A bioassay for evaluating antimalarial activity and for measuring concentration in plasma. Tropical medicine \& international health: TM \& IH. 2, 929-933 https://doi.org/10.1046/j.1365-3156.1997.d01-150.x (1997).

80. Ittarat, W. et al. Effects of alpha-thalassemia on pharmacokinetics of the antimalarial agent artesunate. Antimicrob Agents Chemother. 42, 2332-2335 https://doi.org/10.1128/AAC.42.9.2332 (1998).

81. Chhonker, Y. S., Edi, C. \& Murry, D. J. LC-MS/MS method for simultaneous determination of diethylcarbamazine, albendazole and albendazole metabolites in human plasma: Application to a clinical pharmacokinetic study. J Pharm Biomed Anal. 151, 84-90 https://doi.org/10.1016/j.jpba.2017.12.037 (2018).

82. Kaewkhao, K. et al. High sensitivity methods to quantify chloroquine and its metabolite in human blood samples using LC-MS/MS. Bioanalysis. 11, 333-347 https://doi.org/10.4155/bio-2018-0202 (2019). 
83. Carrara, V. I. et al. Changes in the treatment responses to artesunate-mefloquine on the northwestern border of Thailand during 13 years of continuous deployment. PLoS One. 4, e4551 https://doi.org/10.1371/journal.pone.0004551 (2009).

84. Saunders, D. L. et al. Dihydroartemisinin-piperaquine failure in Cambodia. The New England journal of medicine. 371, 484-485 https://doi.org/10.1056/NEJMc1403007 (2014).

85. Shrestha, B. et al. Distribution and temporal dynamics of P. falciparum chloroquine resistance transporter mutations associated with piperaquine resistance in Northern Cambodia. The Journal of infectious diseases. https://doi.org/10.1093/infdis/jiab055 (2021)

\section{Figures}

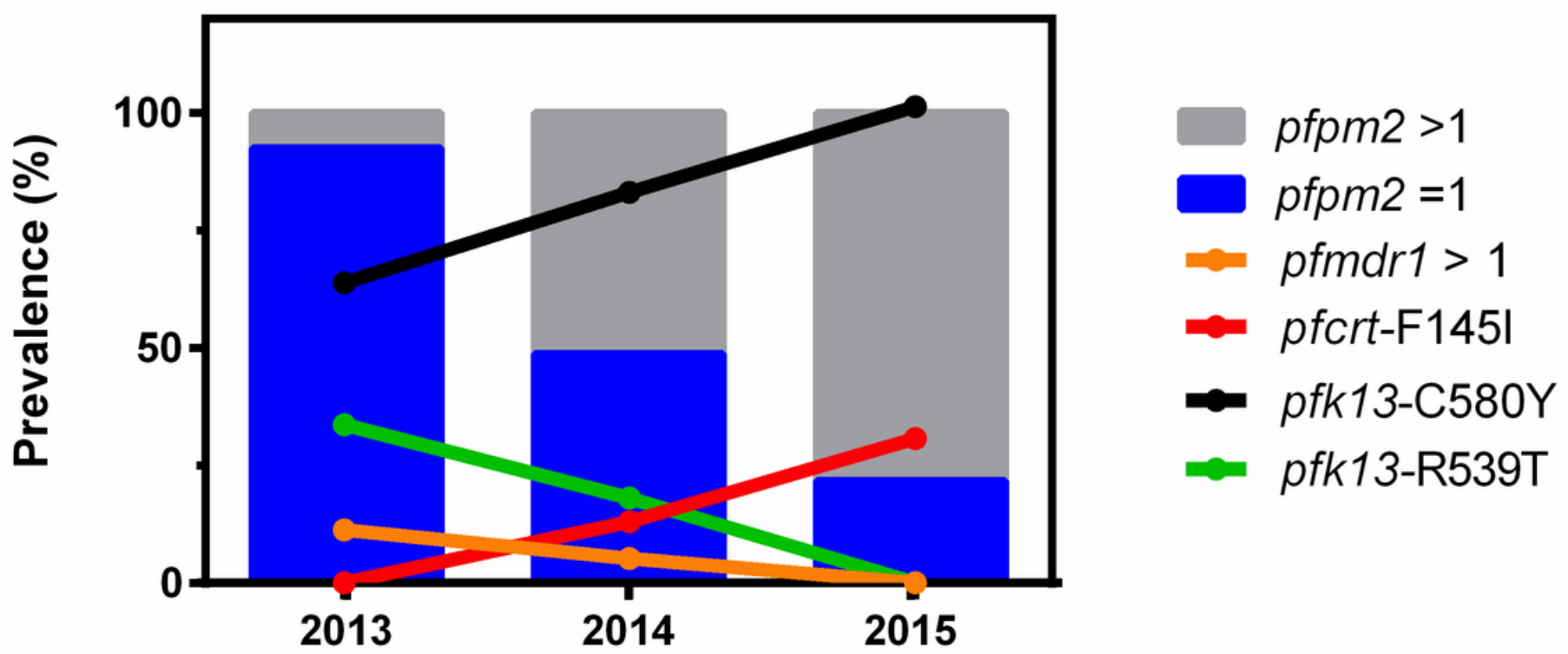

\section{Figure 1}

Prevalence of antimalarial drug resistance mutations over time in Thailand from 2013-2015. Bars indicate the prevalence of parasites with single (blue) and amplified (grey) pfpm2 copy number as a marker of PPQ resistance. The red line indicates the prevalence of parasites harboring pfcrt-F145I mutation associated with PPQ resistance. The orange line indicates the prevalence of amplified pfmdr1 copy number, as a marker of MQ resistance. The black and green lines indicate the prevalence of parasites harboring pfk $13-\mathrm{C} 580 \mathrm{Y}$ and pfk13-R539T mutations as markers of ART resistance. Noted that the national treatment guidelines in Thailand from 2013 to 2015 recommended AS-MQ for P. falciparum malaria 
A
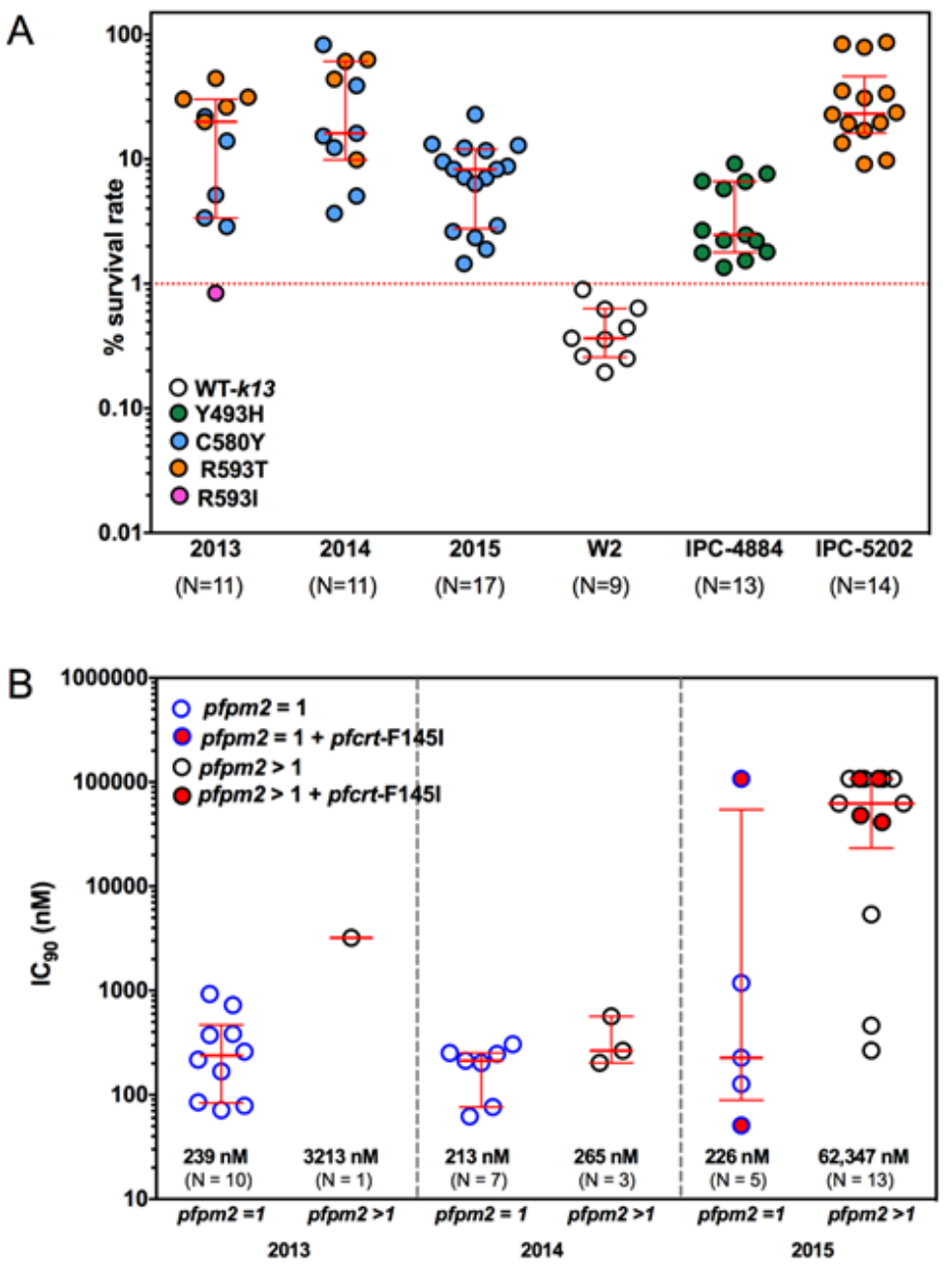

Figure 2

In vitro characterization. (A) RSA and pfk13 mutations. RSA0-3h survival rate for standard laboratory-adapted clones (W2 for ARTsensitive control, IPC-4884 and IPC-5202 for ART-resistance control) and culture-adapted clinical isolates. The dashed line represents the $1 \%$ survival rate cut-off that differentiates ART-resistance $(\geq 1 \%$ red-dashed line) from ART-sensitive $(<1 \%)$ parasites in RSAs. Median and interquartile ranges are shown. White, green, blue, orange, and pink circles represent parasites harboring pfk13-WT, $Y 493 \mathrm{H}$, C580Y, R593T, and R593I, respectively. (B) IC90 to PPQ in parasites with pfcrt-F145I mutation, with and without pfpm2 amplification. Copy number variations of pfpm2 are shown on the $x$-axis, and PPQ IC90 is shown on the $y$-axis (log10 scale). Red circles represent parasites harboring pfcrt-F145I mutation. Bars represent median and interquartile range. 